\title{
On the Stability of a Linear Retarded Differential-Difference Equation
}

\author{
By
}

Hiroyuki NaKaJima

(Kinki University, Japan)

\begin{abstract}
In the present paper, we give a necessary and sufficient condition for the zero solution of a linear retarded system $\mathrm{d} x(t) / \mathrm{d} t=A x(t)+B x(t-\tau)$ to be asymptotically stable. Here $A$ is a real-valued $n \times n$ matrix and $B=b I$, where $b$ is a scalar parameter and $I$ is the $n \times n$ unit matrix.

The stability analysis is reduced to deriving a necessary and sufficient condition for all the roots of a characteristic equation $z-\alpha-\beta \mathrm{e}^{-z}=0$ to have negative real parts. Here $\alpha$ is a complex number defined by $\alpha=\tau \lambda$ with an eigenvalue $\lambda$ of $A$, and $\beta=\tau b$. Our stability criterion is a natural extension of that for the widely-known case where $\alpha$ is a real number.

Key Words and Phrases. Linear retarded differential-difference equation, Stability, Zero solution, Characteristic equation.
\end{abstract}

2010 Mathematics Subject Classification Numbers. 34K06, 34K20.

\section{Introduction}

In the present paper, we study asymptotic stability of the zero solution of a linear retarded system,

$$
\frac{\mathrm{d}}{\mathrm{d} t} x(t)=A x(t)+B x(t-\tau)
$$

Here $x(t)$ is an $n$-dimensional vector, $A$ and $B$ are $n \times n$ real-valued matrices of coefficients and $\tau>0$ is the delay time of the system.

For System (1), Sakata [1] studied the case, $A=a I$, where $a$ is a scalar parameter and $I$ is the $n \times n$ unit matrix, and gave a necessary and sufficient condition for the zero solution of the system to be asymptotically stable. In the present paper, we shall deal with another case, $B=b I$, where $b$ is a scalar parameter. by

In this case, the characteristic function of the system is described

$$
F(s)=\operatorname{det}\left(s I-A-b \mathrm{e}^{-\tau s} I\right)
$$


Let $\lambda_{k}(k=1, \ldots, n)$ be the eigenvalues of the matrix $A$. Then by the similar transformation of matrices, the characteristic equation reduces to

$$
F(s)=\prod_{k=1}^{n}\left(s-\lambda_{k}-b \mathrm{e}^{-\tau s}\right)=0 .
$$

Hence, a necessary and sufficient condition for the zero solution of (1) to be asymptotically stable is that all the roots of the following $n$ equations have negative real parts:

$$
F_{k}(s)=s-\lambda_{k}-b \mathrm{e}^{-\tau s}=0 \quad(k=1, \ldots, n) .
$$

In what follows, we discuss the characteristic equation,

$$
z-\alpha-\beta \mathrm{e}^{-z}=0,
$$

which is derived from each of (2) by transformations, $z=\tau s, \alpha=\tau \lambda_{k}$ and $\beta=\tau b$. This is a widely-known prototype characteristic equation of linear retarded systems, the stability condition of which in the $(\alpha, \beta)$-plane is given by

$$
S=\left\{(\alpha, \beta) \mid \alpha=\frac{v \cos v}{\sin v} \text { and }-\frac{v}{\sin v}<\beta<-\alpha(0<v<\pi)\right\}
$$

for the case where both $\alpha$ and $\beta$ are real [2]. The case where $\alpha$ is real and $\beta$ is complex has also been studied and the stability condition was given [1].

In System (1) that we deal with, $A$ is a general square matrix and $B=b I$. Hence, in what follows, we shall discuss the case where $\alpha$ is complex, $\alpha=p+q \mathrm{i}$, and $\beta$ is real. That is, we consider a necessary and sufficient condition of the set of parameters $(p, q, \beta)$ for all the roots of the following equation to have negative real parts:

$$
f(z)=z-p-q \mathrm{i}-\beta \mathrm{e}^{-z}=0 .
$$

For convenience, we shall call a triplex of values of parameters $(p, q, \beta)$ is stable if all the roots of Eq. (5) have negative real parts.

Braddock and Driessche [3] gave the set of stable parameters on the $(p, q)$ plane with $\beta$ varying. In the present paper, we shall derive this set in the $(p, \beta)$-plane with $q$ varying in $[0, \pi]$ as

$$
S(q)=\left\{(p, \beta) \mid p<-\beta \cos v \text { and } \beta=\frac{q-v}{\sin v}(0<v<\pi)\right\},
$$

which is more natural extension of (4) to the case where $\alpha$ is complex. Although the range of $q$ is restricted to $[0, \pi]$ here, that is sufficient and the reason will be clarified in the beginning of the next section. 


\section{Main results}

In order to derive the stability condition of the zero solution of Eq. (1), we shall first show that it is sufficient to consider only the case where the range of the parameter $q$ in (5) is restricted to $0 \leq q \leq \pi$.

The complex conjugate of the characteristic equation (5) is

$$
w-p+q \mathrm{i}-\beta \mathrm{e}^{-w}=0,
$$

where $w=\bar{z}$. Therefore, $z$ is a root of (5) if and only if $w$ is a root of (6). That is, since $\operatorname{Re}(z)=\operatorname{Re}(w)$, if $(p, q, \beta)$ is stable, then $(p,-q, \beta)$ is also stable.

Furthermore, if $q>\pi$ then there exists an integer $m$ such that $\tilde{q}=q-2 m \pi$ is in the interval $[-\pi, \pi]$. Hence the characteristic equation (5) becomes

$$
z-p-(\tilde{q}+2 m \pi) \mathrm{i}-\beta \mathrm{e}^{-z}=0 .
$$

With a new variable $\tilde{z}=z-2 m \pi \mathrm{i}$, this is transformed to an equation of $\tilde{z}$ as

$$
\tilde{z}-p-\tilde{q} \mathrm{i}-\beta \mathrm{e}^{-\tilde{z}-2 m \pi \mathrm{i}}=\tilde{z}-p-\tilde{q} \mathrm{i}-\beta \mathrm{e}^{-\tilde{z}}=0 .
$$

Obviously $\operatorname{Re}(z)=\operatorname{Re}(\tilde{z})$, hence $(p, q, \beta)$ is stable if and only if $(p, \tilde{q}, \beta)$ is stable.

Consequently, the dependence on the parameters $(p, q, \beta)$ of the stability of characteristic roots for (5) can be described in the space of $(p, \check{q}, \beta)$. Here $\check{q}$ is defined by

$$
\check{q}= \begin{cases}\hat{q} & (0 \leq \hat{q} \leq \pi), \\ 2 \pi-\hat{q} & (\pi<\hat{q}<2 \pi),\end{cases}
$$

where $\hat{q}=|q| \bmod 2 \pi$.

In what follows, we shall discuss the region of the $(p, \beta)$-plane in which any $(p, q, \beta)$ is stable, for each fixed $q$ in the interval $[0, \pi]$. We call this region the stability region.

First, we shall consider the cases where $q=0$ and $q=\pi$. The following lemma gives the stability criteria:

Lemma 1. In case $q=0$, the stability region of $E q$. (5) is given by

$$
S(0)=\left\{(p, \beta) \mid p=\frac{v \cos v}{\sin v} \text { and }-\frac{v}{\sin v}<\beta<-p(0<v<\pi)\right\},
$$

whereas when $q=\pi$, that is given by

$$
S(\pi)=\left\{(p, \beta) \mid p=\frac{v \cos v}{\sin v} \text { and } p<\beta<\frac{v}{\sin v}(0<v<\pi)\right\} .
$$

Proof. In case $q=0$, the characteristic equation (5) is nothing but the prototype equation (3) with $\alpha$ replaced with $p$. Hence (8) is obviously derived from (4). 
For the case $q=\pi$, let $\tilde{z}=z-\pi \mathrm{i}$. Then it is easy to see that (5) can be written by an equation of $\tilde{z}$ as

$$
\tilde{z}-p+\beta \mathrm{e}^{-\tilde{z}}=0 .
$$

This is Eq. (3) except that $\alpha$ is replaced with $p$ and the sign of $\beta$ is opposite. Hence, by noting that $\operatorname{Re}(z)=\operatorname{Re}(\tilde{z})$, we can directly obtain the stability region (9) from (4).

In case $0<q<\pi$, the following lemma gives the stability region:

Lemma 2. In case $0<q<\pi$, the stability region of $E q$. (5) is given by

$$
S(q)=\left\{(p, \beta) \mid p<-\beta \cos v \text { and } \beta=\frac{q-v}{\sin v}(0<v<\pi)\right\} .
$$

Proof. Letting $z=\mu+v$ i, we obtain the following two equations from (5).

$$
\begin{gathered}
\mu-p-\beta \mathrm{e}^{-\mu} \cos v=0, \\
v-q+\beta \mathrm{e}^{-\mu} \sin v=0 .
\end{gathered}
$$

First, when $\beta=0,(5)$ is the characteristic equation of a one-dimensional ordinary differential equation, $\mathrm{d} x / \mathrm{d} t=\alpha x$, with a complex coefficient $\alpha=$ $p+q$ i. Since the real part of its characteristic root is $\mu=p$, the set,

$$
S_{B}=\{(p, \beta) \mid p<0 \text { and } \beta=0\},
$$

a half line on the $p$-axis in the $(p, \beta)$-plane, is clearly included in the stability region. We shall call this set $S_{B}$ the basic stability set.

If the real part of one of the characteristic roots becomes positive after the pair of parameters $(p, \beta)$ varies from $S_{B}$, the root must cross the imaginary axis from left to right in the complex plane at some value of $(p, \beta)$. The values of $(p, \beta)$ such that one of the characteristic roots is on the imaginary axis satisfy the following equation:

$$
\begin{array}{r}
-p-\beta \cos v=0, \\
v-q+\beta \sin v=0,
\end{array}
$$

which is obtained from (11) and (12) by letting $\mu=0$.

Here, suppose $\sin v=0$, then we get $v=q$ from (15), which clearly contradicts our assumption that $0<q<\pi$. Hence the set of values of $(p, \beta)$ such that one of the characteristic roots is on the imaginary axis is given as a family of curves that satisfy (14) and (15) with $v$ varying in $(-\infty,+\infty)$ except the values $v=m \pi$ for any integer $m$.

Therefore, investigating the direction in which one of the characteristic roots crosses the imaginary axis when $(p, \beta)$ varies from the basic stability set $S_{B}$ 
defined by (13), and crosses the curves defined by (14) and (15), we can find the whole stability region in the $(p, \beta)$-plane.

From (14) and (15), we can describe $p$ and $\beta$ as functions of $v$,

$$
\begin{aligned}
& p(v)=-\frac{(q-v) \cos v}{\sin v}, \\
& \beta(v)=\frac{q-v}{\sin v} .
\end{aligned}
$$

The derivatives of these functions are calculated as follows:

$$
\begin{aligned}
& \frac{\mathrm{d} p}{\mathrm{~d} v}=\frac{\cos v \sin v+q-v}{\sin ^{2} v}, \\
& \frac{\mathrm{d} \beta}{\mathrm{d} v}=-\frac{\sin v+(q-v) \cos v}{\sin ^{2} v} .
\end{aligned}
$$

Or from (16) and (17) we have

$$
\begin{aligned}
& \frac{\mathrm{d} p}{\mathrm{~d} v}=\frac{\cos v+\beta}{\sin v}, \\
& \frac{\mathrm{d} \beta}{\mathrm{d} v}=\frac{p-1}{\sin v} .
\end{aligned}
$$

In what follows, we shall determine whether the real part of a characteristic root $z$ changes from positive to negative, or vice versa, when $(p, \beta)$ crosses the curves defined by (16) and (17).

First, we shall consider the characteristic root $z$ to be a function of $p$ with $\beta$ and $q$ fixed, and calculate its derivative,

$$
\left.\frac{\mathrm{d} \mu}{\mathrm{d} p}\right|_{\mu=0}=\left.\operatorname{Re}\left(\frac{\mathrm{d} z}{\mathrm{~d} p}\right)\right|_{\mu=0} .
$$

Since $z=z(p)$ is the characteristic root of $(5)$, that is $f(z(p))=0$ for all $p$, we have

$$
\frac{\mathrm{d} f}{\mathrm{~d} p}=\frac{\partial f}{\partial z} \frac{\mathrm{d} z}{\mathrm{~d} p}+\frac{\partial f}{\partial p}=0 .
$$

Here,

$$
\frac{\partial f}{\partial z}=1+\beta \mathrm{e}^{-z}=1+\beta \mathrm{e}^{-\mu}(\cos v-\mathrm{i} \sin v) .
$$

Hence,

$$
\left.\frac{\partial f}{\partial z}\right|_{\mu=0}=1+\beta(\cos v-\mathrm{i} \sin v) .
$$


Since $\sin v \neq 0$ as we assumed above, it is easy to see that $1+\beta(\cos v-\mathrm{i} \sin v)$ $\neq 0$. Therefore $\left.(\partial f / \partial z)\right|_{\mu=0} \neq 0$, and hence from $(21),\left.(\mathrm{d} z / \mathrm{d} p)\right|_{\mu=0}$ can be calculated by

$$
\left.\frac{\mathrm{d} z}{\mathrm{~d} p}\right|_{\mu=0}=-\left.\left(\frac{\partial f}{\partial p} / \frac{\partial f}{\partial z}\right)\right|_{\mu=0} .
$$

Consequently, we obtain

$$
\begin{aligned}
\left.\frac{\mathrm{d} \mu}{\mathrm{d} p}\right|_{\mu=0} & =\left.\operatorname{Re}\left(\frac{\mathrm{d} z}{\mathrm{~d} p}\right)\right|_{\mu=0} \\
& =\operatorname{Re}\left(-\left.\left(\frac{\partial f}{\partial p} / \frac{\partial f}{\partial z}\right)\right|_{\mu=0}\right) \\
& =\left.\operatorname{Re}\left(\frac{1}{1+\beta \mathrm{e}^{-z}}\right)\right|_{\mu=0} \\
& =\left.\operatorname{Re}\left(\frac{1}{1+\beta \mathrm{e}^{-\mu}(\cos v-\mathrm{i} \sin v)}\right)\right|_{\mu=0} \\
& =\frac{1+\beta \cos v}{(1+\beta \cos v)^{2}+\beta^{2} \sin ^{2} v}
\end{aligned}
$$

In a similar way, when we consider the characteristic root $z$ to be a function of $\beta$ with $p$ and $q$ fixed, we also obtain

$$
\begin{aligned}
\left.\frac{\mathrm{d} \mu}{\mathrm{d} \beta}\right|_{\mu=0} & =\left.\operatorname{Re}\left(\frac{\mathrm{d} z}{\mathrm{~d} \beta}\right)\right|_{\mu=0} \\
& =\operatorname{Re}\left(-\left.\left(\frac{\partial f}{\partial \beta} / \frac{\partial f}{\partial z}\right)\right|_{\mu=0}\right) \\
& =\left.\operatorname{Re}\left(\frac{\mathrm{e}^{-z}}{1+\beta \mathrm{e}^{-z}}\right)\right|_{\mu=0} \\
& =\left.\operatorname{Re}\left(\frac{\mathrm{e}^{-\mu}(\cos v-\mathrm{i} \sin v)}{1+\beta \mathrm{e}^{-\mu}(\cos v-\mathrm{i} \sin v)}\right)\right|_{\mu=0} \\
& =\frac{\cos v+\beta}{(1+\beta \cos v)^{2}+\beta^{2} \sin ^{2} v}
\end{aligned}
$$

Now we consider the shape and location of curves $(p(v), \beta(v))$ defined by (16) and (17), at which the characteristic root is on the imaginary axis, and the direction in which the root crosses the axis. We shall divide the range of the imaginary part $v$ of the characteristic root into the following five parts. 
Case I: $0<v<\pi$

From (16) and (17), we obtain the following relations:

$$
\begin{aligned}
p(q) & =0, \quad \beta(q)=0, \\
\lim _{v \rightarrow 0+0} p(v) & =\lim _{v \rightarrow 0+0}\left[\frac{-(q-v) \cos v}{\sin v}\right]=-\infty, \\
\lim _{v \rightarrow 0+0} \beta(v) & =\lim _{v \rightarrow 0+0} \frac{q-v}{\sin v}=+\infty, \\
\lim _{v \rightarrow \pi-0} p(v) & =\lim _{v \rightarrow \pi-0}\left[\frac{-(q-v) \cos v}{\sin v}\right]=-\infty, \\
\lim _{v \rightarrow \pi-0} \beta(v) & =\lim _{v \rightarrow \pi-0} \frac{q-v}{\sin v}=-\infty .
\end{aligned}
$$

Now we shall prove that there is a value $\tilde{v}$ such that $p(\tilde{v})$ is the maximum value of $p(v)$ in $(0, \pi)$, and $p(v)$ is strictly increasing on $(0, \tilde{v})$ and strictly decreasing on $(\tilde{v}, \pi)$, respectively.

Let us define a function $\phi(v)$ by

$$
\phi(v)=\cos v \sin v+q-v .
$$

Since $\phi(0)=q>0$ and $\phi(\pi)=q-\pi<0$, we find that $\phi(v)$ vanishes at some value $\tilde{v}$ by virtue of the intermediate value theorem. Furthermore, $\phi^{\prime}(v)=$ $\cos 2 v-1<0$ for all $v \in(0, \pi)$, hence $\phi(v)$ is positive for all $v \in(0, \tilde{v})$, and negative for all $v \in(\tilde{v}, \pi)$.

Therefore, $p(v)$ is strictly increasing on $(0, \tilde{v})$ and strictly decreasing on $(\tilde{v}, \pi)$, respectively, since $\mathrm{d} p / \mathrm{d} v$ is described by $\mathrm{d} p / \mathrm{d} v=\phi(v) / \sin ^{2} v$ from (18) and (25).

Moreover, since $\mathrm{d} p(\tilde{v}) / \mathrm{d} v=0$, we obtain $q-\tilde{v}=-\sin \tilde{v} \cos \tilde{v}$ from (18). Substituting this relation into (16) we have

$$
p(\tilde{v})=\cos ^{2} \tilde{v}
$$

Therefore $0 \leq p(\tilde{v})<1$ because $\tilde{v} \in(0, \pi)$, and $p(v)<1$ for all $v \in(0, \pi)$ since $p(\tilde{v})$ is the maximum.

On the other hand, from $(20)$ and the fact $p(v)<1$ for all $v \in(0, \pi)$, we have $\mathrm{d} \beta(v) / \mathrm{d} v<0$ for all $v \in(0, \pi)$, that is, $\beta(v)$ is strictly decreasing on this interval.

Consequently, we find that $p$ can be regarded as a function of $\beta, p=p(\beta)$, defined on $(-\infty,+\infty)$. We denote by $C_{I}$ the curve defined by this function. It is easy to see that $C_{I}$ divides the $(p, \beta)$-plane into two regions and that the basic stability set $S_{B}$ lies on the left-hand of $C_{I}$. 
Now we can determine the direction in which the characteristic root crosses the imaginary axis when $(p, \beta)$ crosses the curve $C_{I}$ by calculating $\left.(\mathrm{d} \mu / \mathrm{d} p)\right|_{\mu=0}$ using (22).

From (16) and (17), we get

$$
1+\beta(v) \cos v=1-p(v) .
$$

Besides, as we saw above, $p(v)<1$ for $0<v<\pi$. Therefore, by substituting (27) into (22), we obtain,

$$
\left.\frac{\mathrm{d} \mu}{\mathrm{d} p}\right|_{\mu=0}>0
$$

for all $v \in(0, \pi)$. This implies that when $(p, \beta)$ crosses the curve $C_{I}$ from left to right on the $(p, \beta)$-plane, one of the characteristic roots crosses the imaginary axis from left to right.

Case II: $\quad(2 m-1) \pi<v<2 m \pi ; m=1,2,3, \ldots$

From (16) and (17), we obtain the following relations:

$$
\begin{aligned}
\lim _{v \rightarrow(2 m-1) \pi+0} p(v) & =\lim _{v \rightarrow(2 m-1) \pi+0}\left[\frac{-(q-v) \cos v}{\sin v}\right]=+\infty, \\
\lim _{v \rightarrow(2 m-1) \pi+0} \beta(v) & =\lim _{v \rightarrow(2 m-1) \pi+0} \frac{q-v}{\sin v}=+\infty, \\
\lim _{v \rightarrow 2 m \pi-0} p(v) & =\lim _{v \rightarrow 2 m \pi-0}\left[\frac{-(q-v) \cos v}{\sin v}\right]=-\infty, \\
\lim _{v \rightarrow 2 m \pi-0} \beta(v) & =\lim _{v \rightarrow 2 m \pi-0} \frac{q-v}{\sin v}=+\infty .
\end{aligned}
$$

It follows from $(25)$ that $\phi((2 m-1) \pi)=q-(2 m-1) \pi<0$ for every positive integer $m$ since $0<q<\pi$. We can also see $\mathrm{d} \phi(v) / \mathrm{d} v=\cos 2 v-1$ $<0$ for all $v \in((2 m-1) \pi, 2 m \pi)$. Hence, from (18) and (25), we can see $\mathrm{d} p(v) / \mathrm{d} v<0$ holds for all $v \in((2 m-1) \pi, 2 m \pi)$ and for every positive integer $m$. That is, $p(v)$ is strictly decreasing on all these intervals $((2 m-1) \pi, 2 m \pi)$.

From (28), (29) and the fact that $p(v)$ is strictly decreasing, we see that there exists exactly one value $\bar{v}$ that satisfies $p(\bar{v})=1$ for each interval $((2 m-1) \pi, 2 m \pi)$.

Hence, from (20) and the fact that $\sin v<0$ in these intervals, we find that $\beta(v)$ is strictly decreasing on $((2 m-1) \pi, \bar{v})$, and strictly increasing on $(\bar{v}, 2 m \pi)$.

Consequently, we find that $\beta$ is a function of $p, \beta=\beta(p)$, defined on $(-\infty,+\infty)$, which attains its minimum at $p=p(\bar{v})=1$. We denote by $C_{I I}(m)$ the curve defined by $\beta(p)$ for $v \in((2 m-1) \pi, 2 m \pi)$. Each of the curves $C_{I I}(m)$ 
divides the $(p, \beta)$-plane into two regions, and the basic set $S_{B}$ lies on the downside of each $C_{I I}(m)$ since $(17)$ implies $\beta(v)>0$ for all $v \in((2 m-1) \pi, 2 m \pi)$.

Comparing (19),

$$
\frac{\mathrm{d} p}{\mathrm{~d} v}=\frac{\cos v+\beta}{\sin v}
$$

with (23),

$$
\left.\frac{\mathrm{d} \mu}{\mathrm{d} \beta}\right|_{\mu=0}=\frac{\cos v+\beta}{(1+\beta \cos v)^{2}+\beta^{2} \sin ^{2} v},
$$

and noticing that $\sin v<0$ and $\mathrm{d} p(v) / \mathrm{d} v<0$, we can conclude the following inequality holds for all $v \in((2 m-1) \pi, 2 m \pi)$ and for every positive integer $m$ :

$$
\left.\frac{\mathrm{d} \mu}{\mathrm{d} \beta}\right|_{\mu=0}>0 .
$$

This implies that when $(p, \beta)$ crosses each of $C_{I I}(m)$ upward on the $(p, \beta)$-plane, one of the characteristic roots crosses the imaginary axis from left to right.

Case III: $\quad-2 m \pi<v<-(2 m-1) \pi ; m=1,2,3, \ldots$

From (16) and (17), we obtain the following relations:

$$
\begin{aligned}
\lim _{v \rightarrow-2 m \pi+0} p(v) & =\lim _{v \rightarrow-2 m \pi+0}\left[\frac{-(q-v) \cos v}{\sin v}\right]=-\infty, \\
\lim _{v \rightarrow-2 m \pi+0} \beta(v) & =\lim _{v \rightarrow-2 m \pi+0} \frac{q-v}{\sin v}=+\infty, \\
\lim _{v \rightarrow-(2 m-1) \pi-0} p(v) & =\lim _{v \rightarrow-(2 m-1) \pi-0}\left[\frac{-(q-v) \cos v}{\sin v}\right]=+\infty, \\
\lim _{v \rightarrow-(2 m-1) \pi-0} \beta(v) & =\lim _{v \rightarrow-(2 m-1) \pi-0} \frac{q-v}{\sin v}=+\infty .
\end{aligned}
$$

By almost the same arguments as in Case II, we find that $p(v)$ is strictly increasing on $(-2 m \pi,-(2 m-1) \pi)$, and $\beta(v)$ is strictly decreasing on $(-2 m \pi, \bar{v})$, and strictly increasing on $(\bar{v},-(2 m-1) \pi)$, where $\bar{v}$ is a certain value such that $p(\bar{v})=1$.

Consequently, we find that $\beta$ is a function of $p, \beta=\beta(p)$, defined on $(-\infty,+\infty)$, which attains its minimum at $p=p(\bar{v})=1$. We denote by $C_{I I I}(m)$ the curve defined by $\beta(p)$ for $v \in(-2 m \pi,-(2 m-1) \pi)$. Each of the curves $C_{I I I}(m)$ divides the $(p, \beta)$-plane into two regions, and the basic set $S_{B}$ lies on the down-side of each $C_{I I I}(m)$ since $\beta(v)>0$ for all $v \in(-2 m \pi,-(2 m-1) \pi)$.

Comparing (19) with (23), and noticing that $\sin v>0$ and $\mathrm{d} p(v) / \mathrm{d} v>0$, we can conclude the following inequality holds for all $v \in(-2 m \pi,-(2 m-1) \pi)$ and 
for every positive integer $m$ :

$$
\left.\frac{\mathrm{d} \mu}{\mathrm{d} \beta}\right|_{\mu=0}>0 .
$$

This implies that when $(p, \beta)$ crosses each of $C_{I I I}(m)$ upward on the $(p, \beta)$ plane, one of the characteristic roots crosses the imaginary axis from left to right.

Case IV: $2 m \pi<v<(2 m+1) \pi ; m=1,2,3, \ldots$

From (16) and (17), we obtain the following relations:

$$
\begin{aligned}
\lim _{v \rightarrow 2 m \pi+0} p(v) & =\lim _{v \rightarrow 2 m \pi+0}\left[\frac{-(q-v) \cos v}{\sin v}\right]=+\infty, \\
\lim _{v \rightarrow 2 m \pi+0} \beta(v) & =\lim _{v \rightarrow 2 m \pi+0} \frac{q-v}{\sin v}=-\infty, \\
\lim _{v \rightarrow(2 m+1) \pi-0} p(v) & =\lim _{v \rightarrow(2 m+1) \pi-0}\left[\frac{-(q-v) \cos v}{\sin v}\right]=-\infty, \\
\lim _{v \rightarrow(2 m+1) \pi-0} \beta(v) & =\lim _{v \rightarrow(2 m+1) \pi-0} \frac{q-v}{\sin v}=-\infty .
\end{aligned}
$$

By almost the same arguments as in Case II, we find that $p(v)$ is strictly decreasing on $(2 m \pi,(2 m+1) \pi)$, and $\beta(v)$ is strictly increasing on $(2 m \pi, \bar{v})$, and strictly decreasing on $(\bar{v},(2 m+1) \pi)$, where $\bar{v}$ is a certain value such that $p(\bar{v})=1$.

Consequently, we find that $\beta$ is a function of $p, \beta=\beta(p)$, defined on $(-\infty,+\infty)$, which attains its maximum at $p=p(\bar{v})=1$. We denote by $C_{I V}(m)$ the curve defined by $\beta(p)$ for $v \in(2 m \pi,(2 m+1) \pi)$. Each of the curves $C_{I V}(m)$ divides the $(p, \beta)$-plane into two regions, and the basic set $S_{B}$ lies on the upperside of each $C_{I V}(m)$ since $\beta(v)<0$ for all $v \in(2 m \pi,(2 m+1) \pi)$.

Comparing (19), with (23), and noticing that $\sin v>0$ and $\mathrm{d} p(v) / \mathrm{d} v<0$, we can conclude the following inequality holds for all $v \in(2 m \pi,(2 m+1) \pi)$ and for every positive integer $m$ :

$$
\left.\frac{\mathrm{d} \mu}{\mathrm{d} \beta}\right|_{\mu=0}<0 .
$$

This implies that when $(p, \beta)$ crosses each of $C_{I V}(m)$ downward on the $(p, \beta)$ plane, one of the characteristic roots crosses the imaginary axis from left to right.

Case V: $\quad-(2 m+1) \pi<v<-2 m \pi ; m=1,2,3, \ldots$

From (16) and (17), we obtain the following relations: 


$$
\begin{aligned}
\lim _{v \rightarrow-(2 m+1) \pi+0} p(v) & =\lim _{v \rightarrow-(2 m+1) \pi+0}\left[\frac{-(q-v) \cos v}{\sin v}\right]=-\infty, \\
\lim _{v \rightarrow-(2 m+1) \pi+0} \beta(v) & =\lim _{v \rightarrow-(2 m+1) \pi+0} \frac{q-v}{\sin v}=-\infty, \\
\lim _{v \rightarrow-2 m \pi-0} p(v) & =\lim _{v \rightarrow-2 m \pi-0}\left[\frac{-(q-v) \cos v}{\sin v}\right]=+\infty, \\
\lim _{v \rightarrow-2 m \pi-0} \beta(v) & =\lim _{v \rightarrow-2 m \pi-0} \frac{q-v}{\sin v}=-\infty .
\end{aligned}
$$

By almost the same arguments as in Case II, we find that $p(v)$ is strictly increasing on $(-(2 m+1) \pi,-2 m \pi)$, and $\beta(v)$ is strictly increasing on $(-(2 m+1) \pi, \bar{v})$, and strictly decreasing on $(\bar{v},-2 m \pi)$, where $\bar{v}$ is a certain value such that $p(\bar{v})=1$.

Consequently, we find that $\beta$ is a function of $p, \beta=\beta(p)$, defined on $(-\infty,+\infty)$, which attains its maximum at $p=p(\bar{v})=1$. We denote by $C_{V}(m)$ the curve defined by $\beta(p)$ for $v \in(-(2 m+1) \pi,-2 m \pi)$. Each of the curves $C_{V}(m)$ divides the $(p, \beta)$-plane into two regions, and the basic set $S_{B}$ lies on the upper-side of each $C_{V}(m)$ since $\beta(v)<0$ for all $v \in(-(2 m+1) \pi,-2 m \pi)$.

Comparing (19), with (23), and noticing that $\sin v<0$ and $\mathrm{d} p(v) / \mathrm{d} v>0$, we can conclude the following inequality holds for all $v \in(-(2 m+1) \pi,-2 m \pi)$ and for every positive integer $m$ :

$$
\left.\frac{\mathrm{d} \mu}{\mathrm{d} \beta}\right|_{\mu=0}<0 .
$$

This implies that when $(p, \beta)$ crosses each of $C_{V}(m)$ downward on the $(p, \beta)$ plane, one of the characteristic roots crosses the imaginary axis from left to right.

From the five cases considered above, we can conclude that if the parameter pair $(p, \beta)$ varies from the basic set $S_{B}$ defined by (13), and crosses each of the curves $C_{I}, C_{I I}(m), C_{I I I}(m), C_{I V}(m)$ and $C_{V}(m)$, then one of the stable characteristic roots always crosses the imaginary axis from left to right.

Finally, in order to complete our proof, we shall show that $C_{I}$ does not intersect with any of other curves, $C_{I I}(m), C_{I I I}(m), C_{I V}(m)$ and $C_{V}(m)$, for any positive integer $m$.

Suppose $(p(v), \beta(v))=\left(p\left(v^{\prime}\right), \beta\left(v^{\prime}\right)\right)$ for some $v$ and $v^{\prime}$ such that $v \in(0, \pi)$ and $v^{\prime} \notin(0, \pi)$, then from (16) and (17) we obtain,

$$
\begin{aligned}
\beta(v) & =\beta\left(v^{\prime}\right), \\
\beta(v) \cos v & =\beta\left(v^{\prime}\right) \cos v^{\prime} .
\end{aligned}
$$


If $\beta(v)=\beta\left(v^{\prime}\right)=0$ then we find $v=v^{\prime}=q$ from (17), which contradicts $q \in(0, \pi)$ and $v^{\prime} \notin(0, \pi)$. Thus we can assume $\beta(v)=\beta\left(v^{\prime}\right) \neq 0$ and hence from $(31)$ we find

$$
\cos v=\cos v^{\prime}
$$

First, let us consider the case where $v^{\prime}$ is in the interval for Case II or Case V. From (32) we find that $v+v^{\prime}=2 m \pi$ for some non-zero integer $m$, and hence $\sin v=-\sin v^{\prime}$. Therefore, from (17) and (30) we obtain $q-v=v^{\prime}-q$ and hence $q=\left(v+v^{\prime}\right) / 2$. However, since $v+v^{\prime}=2 m \pi$ as shown above, $q=m \pi$ holds for some non-zero integer $m$, which contradicts $q \in(0, \pi)$.

On the other hand, for the case where $v^{\prime}$ is in the interval for Case III or Case IV, from (32) we find that $v-v^{\prime}=2 m \pi$ for some non-zero integer $m$, and hence $\sin v=\sin v^{\prime}$. Therefore, from (17) and (30) we obtain $v=v^{\prime}$. That clearly contradicts the assumption $v \in(0, \pi)$ and $v^{\prime} \notin(0, \pi)$.

Consequently, we find that $C_{I}$ does not intersect with any of other curves, $C_{I I}(m), C_{I I I}(m), C_{I V}(m)$ and $C_{V}(m)$, for every positive integer $m$.

Since the curve $C_{I}$ touches the basic stability set $S_{B}$ at $(p, \beta)=(0,0)$ as is shown in (24), $C_{I}$ is the nearest to $S_{B}$ among $C_{I}, C_{I I}(m), C_{I I I}(m), C_{I V}(m)$ and $C_{V}(m)$. That implies that $C_{I}$ is the boundary of the stability region, and hence the region is described by the set $S(q)$ defined by (10). Thus the proof is completed.

The following theorem immediately follows from the two lemmas above.

Theorem. Let us denote by $\lambda_{k}(k=1, \ldots, n)$ the eigenvalues of the matrix $A$ in (1). Then the zero solution of (1) with $B=b I$ is asymptotically stable if and only if

$$
\left(p_{k}, \beta\right) \in S\left(\check{q}_{k}\right)
$$

holds for all $k=1, \ldots, n$. Here $p_{k}=\operatorname{Re}\left(\tau \lambda_{k}\right), \beta=\tau b, \check{q}_{k}$ is defined by (7) for $q_{k}=\operatorname{Im}\left(\tau \lambda_{k}\right)$, and $S(q)$ is defined by (8), (9) and (10) for $q \in[0, \pi]$.

Remark. The relationship between Lemmas 1 and 2 is explained as follows:

First, since the maximum point $\tilde{v}$ of $p(v)$ defined on $(0, \pi)$ satisfies $q-\tilde{v}=$ $-(1 / 2) \sin 2 \tilde{v}$, it is easy to see that $\tilde{v}>q$ if $q \in(0, \pi / 2)$, and that $\tilde{v} \rightarrow 0$ when $q \rightarrow 0$. From (26) we can see $p(\tilde{v})=\cos ^{2} \tilde{v}$ and $\beta(\tilde{v})=-p(\tilde{v}) / \cos \tilde{v}=-\cos \tilde{v}$. Therefore if $q>0$ is sufficiently small then $\tilde{v} \approx 0$, hence we obtain the following approximation of $(p(v), \beta(v))$ that describes the curve $C_{I}$ : 
(33) $(p(v), \beta(v))= \begin{cases}(-\cos v \beta(v), \beta(v)) \approx(-\beta(v), \beta(v)) & (0<v<\tilde{v}), \\ \left(\cos ^{2} \tilde{v},-\cos \tilde{v}\right) \approx(1,-1), & (v=\tilde{v}), \\ \left(-\frac{(q-v) \cos v}{\sin v}, \frac{q-v}{\sin v}\right) \approx\left(\frac{v \cos v}{\sin v},-\frac{v}{\sin v}\right) & (\tilde{v}<v<\pi) .\end{cases}$

On the other hand, we can see that the stability region $S(0)$ for $q=0$ given by (8) can also be described as follows:

$$
S(0)=\{(p, \beta) \mid p<\psi(\beta)\},
$$

where the function $\psi$ is defiend by

$$
\psi(\beta)= \begin{cases}-\beta \cos v(\beta) & (\beta<-1) \\ -\beta & (\beta \geq-1),\end{cases}
$$

and $v(\beta)$ is the inverse function of $\beta(v)=-v / \sin v$ on $(0, \pi)$.

Consequently, from (33), (34) and (35), we find that the boundary of the stability region $S(q)$, that is, the curve $C_{I}$ converges to that of $S(0)$ when $q \rightarrow 0$.

In a similar way, we can see that if $q$ is sufficiently close to $\pi$, the curve $C_{I}$ is approximately described by

$$
\begin{aligned}
& (p(\omega), \beta(\omega)) \\
& \quad= \begin{cases}(\cos \omega \beta(\omega), \beta(\omega)) \approx(\beta(\omega), \beta(\omega)) & (0<\omega<\tilde{\omega}), \\
\left(\cos ^{2} \tilde{\omega}, \cos \tilde{\omega}\right) \approx(1,1), & (\omega=\tilde{\omega}), \\
\left(-\frac{\left(q^{\prime}-\omega\right) \cos \omega}{\sin \omega},-\frac{q^{\prime}-\omega}{\sin \omega}\right) \approx\left(\frac{\omega \cos \omega}{\sin \omega}, \frac{\omega}{\sin \omega}\right) & (\tilde{\omega}<\omega<\pi),\end{cases}
\end{aligned}
$$

where $q^{\prime}=\pi-q, \omega=\pi-v$ and $\tilde{\omega}=\pi-\tilde{v}$. We also obtain another description of the stability region $S(\pi)$,

$$
S(\pi)=\{(p, \beta) \mid p<\chi(\beta)\},
$$

where the function $\chi$ is defiend by

$$
\chi(\beta)= \begin{cases}\beta & (\beta \leq 1) \\ \beta \cos \omega(\beta) & (\beta>1),\end{cases}
$$

and $\omega(\beta)$ is the inverse function of $\beta(\omega)=\omega / \sin \omega$ on $(0, \pi)$. Thus we find that the curve $C_{I}$ converges to the boundary of $S(\pi)$ when $q \rightarrow \pi$.

Acknowledgement. The author would like to thank Prof. K. Konishi of Osaka Prefecture University for his valuable advice and encouragement. 


\title{
References
}

[1] Sakata, S., Asymptotic Stability for a Linear System of Differential-Difference Equations, Funkcial. Ekvac., 41 (1998), 435-449.

[2 ] Diekmann, O., van Gils, S. A., Verduyn Lunel, S. M. and Walther, H.-O., Delay Equations, Applied Mathematical Sciences, 110, Springer-Verlag, New York, 1995.

[3] Braddock, R. D. and van den Driessche, P., On the Stability of Differential-Difference Equations, J. Austral. Math. Soc. Ser. B, 19 (1976), 358-370.

\author{
nuna adreso: \\ Department of Electronic Engineering and \\ Computer Science \\ Faculty of Engineering \\ Kinki University \\ 1 Takaya Umenobe, Higashi-Hiroshima \\ Hiroshima 739-2116 \\ Japan \\ E-mail: nakajima@hiro.kindai.ac.jp
}

(Ricevita la 9-an de aŭgusto, 2012)

(Reviziita la 20-an de februaro, 2013) 